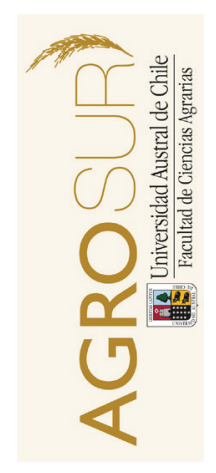

\title{
Influencia de la fecha y densidad de siembra sobre el desarrollo de patógenos presentes en cultivares de haba (Vicia faba L.) de crecimiento determinado
}

\author{
Effect of date and sowing density on the development of pathogens existing in \\ faba bean (Vicia faba L.) cultivars with determinate growth habit.
}

\author{
Doussoulin, $\mathrm{H}^{a *}$, Andrade, N. $^{a}$, Acuña, R. ${ }^{a}$
}

${ }^{a}$ Instituto de Producción y Sanidad Vegetal, Facultad de Ciencias Agrarias, Universidad Austral de Chile.

\begin{tabular}{l} 
A R T I C L E IN F O \\
\hline Article history: \\
Received 18.03.15 \\
Accepted 05.06.15 \\
\hline Keywords: \\
Faba bean pathogens \\
Botrytis fabae \\
B. cinerea \\
Uromyces viciae fabae \\
Cercospora zonata \\
Faba bean \\
\hline Original Research Article, \\
Crop Science \\
\hline *Corresponding author: \\
Herman Doussoulin \\
E-mail address: \\
hdoussoulin@gmail.com
\end{tabular}

\begin{abstract}
A B S T R A C T
Incorporation of new crop alternatives in an area requires to determine the agronomic factors that favour the crop and the potential limiting factors to achieve the potential yield. The objective of this study was to assess the presence and incidence of pathogens and yield according to seeding date, density and cultivar in determinate growth faba bean (Vicia faba L.) cultivars, which has been introduced in the Valdivia area. Presence and incidence of pathogen and yield were evaluated. Assays were conducted at the Santa Rosa Experimental Station and the Laboratory of Plant Pathology of the Universidad Austral de Chile, during two seasons (2009-2010 and 2010-2011). The pathogens found in the crops were Botrytis fabae, B. cinerea, Uromyces viciae fabae and Cercospora zonata. The incidence varied depending on seeding date, during the 2009/2010 season early seeding date (August 7) registered a reduced incidence of B. fabae $(14.1 \%)$ and $U$. viciae fabae $(0.4 \%)$ compared to a late seeding date (September 22) (B. fabae 84.3\%; U. viciae fabae $75.2 \%$ ), while in $2010 / 2011$ season intermediate seeding date registered the lowest incidence of $B$. fabae (45.6\%), with no statistically significant differences being observed for the others pathogens. Plant density and cultivar did not affect the pathogens. Early seeding dates (August 7 and September 1, 2010; September 1, 2011) and high plant density (30 and 40 plants $\mathrm{m}^{-2}$ in the first season and 40 plants $\mathrm{m}^{-2}$ in the second season) had higher yields. Cultivars did not differ in yield. These results suggest that early sowing dates (August to early September) at high densities ( 40 plants $\mathrm{m}^{-2}$ ) should reduce the pathogen incidence and maximize yields of "baby" faba-bean in the Valdivia area.
\end{abstract}

\section{RESUMEN}

La incorporación de nuevas alternativas agrícolas para una zona determinada, conlleva a determinar qué factores agronómicos son favorables, y cuáles pueden ser limitantes para alcanzar el rendimiento potencial. El objetivo de la presente investigación, fue evaluar la presencia e incidencia de patógenos en función de la fecha y densidad de siembra sobre cultivares de haba de crecimiento determinado introducidos en la zona de Valdivia. Los ensayos se realizaron en la Estación Experimental Agropecuaria Austral y el Laboratorio de Fitopatología de la Universidad Austral de Chile, durante dos temporadas (2009-2010 y 2010-2011). Los patógenos que se presentaron en el cultivo fueron, Botrytis fabae, B. cinerea, Uromyces viciae fabae y Cercospora zonata. La incidencia varió dependiendo del momento de siembra, durante la temporada 2009/2010 la siembra temprana (7 de agosto) presentó una menor incidencia de B. fabae (14,1\%), y U. viciae fabae $(0,4 \%)$ comparado a fechas tardías de siembra $(22$ de septiembre) (B. fabae 84,3\%, U. viciae fabae 75,2\%), para la temporada 2010/2011, la fecha de siembra intermedia presentó la menor incidencia de $B$. fabae $(45,6 \%$ ) y para los demás patógenos no se observaron diferencias estadísticamente significativas. La densidad de siembra y cultivar no influyeron en la incidencia de patógenos. Siembras tempranas (7 de agosto y 1 de septiembre de 2010; 1 de septiembre de 2011) y altas densidades de siembra (30 y 40 plantas $\mathrm{m}^{-2}$, para la primera temporada y 40 plantas $\mathrm{m}^{-2}$, para la segunda) alcanzaron los mayores rendimientos. No se observó diferencias de rendimiento entre cultivares. Esto

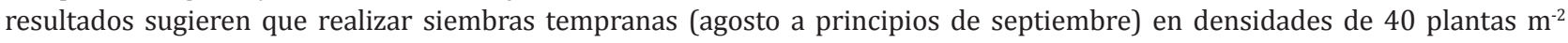
presentarán una menor incidencia de patógenos y maximizarán los rendimientos de haba "baby" en la zona de Valdivia.

Palabras clave: Patógenos haba, Botrytis fabae, B. cinerea, Uromyces viciae fabae, Cercospora zonata, haba.

\section{INTRODUCCIÓN}

El haba (Vicia faba L.) es una planta leguminosa anual, cuyo uso principal es la utilización de granos y vainas en alimentación humana y animal, destacan- do por su alto contenido de proteína (Faiguenbaum, 2003). La producción de este cultivo en Chile se concentra principalmente en la zona centro del país, siendo muy poco explotada en la zona sur, a pesar de que existen las condiciones agroecológicas apropiadas 
para producirlas. Con el fin de desarrollar el sector hortícola de la zona, se ha planteado la incorporación de habas tipo "baby", de crecimiento determinado y aptitud agroindustrial. La ventaja que presentan estos cultivares en relación a los tradicionalmente utilizados (crecimiento indeterminado), es que presentan menor variabilidad del producto cosechado y además son aptas para cosecha mecánica, esto dado a que la floración es homogénea y las vainas se encuentran concentradas en la parte apical de las plantas las cuales no superan los $90 \mathrm{~cm}$. (Nadal et al., 2004). Sin embargo, al introducir un nuevo cultivar en una zona, es fundamental analizar cuáles son los principales patógenos que pueden afectar al cultivo, siendo las enfermedades de tipo fungosas las más importantes para esta especie, destacando la mancha chocolate (Botrytis fabae Sard.), cercosporiosis (Cercospora zonata Winter), roya del haba (Uromyces viciae fabae Pers.) y antracnosis (Ascochyta fabae Speg.), las cuales pueden afectar notablemente los rendimientos (Hanounik y Bisri, 1991; Amira et al., 2003; Elad et al., 2007; Stoddard et al., 2010). Los niveles de incidencia de una enfermedad pueden ser afectados por factores ambientales como la temperatura y humedad, así como también por factores agronómicos como la fecha de siembra, densidad de plantas o cultivares seleccionados. (López-Bellido et al., 2005), por lo que el objetivo de la presente investigación, fue evaluar la presencia e incidencia de patógenos en función de la fecha y densidad de siembra sobre cultivares de haba de crecimiento determinado en la zona de Valdivia.

\section{MATERIALES Y MÉTODOS}

\section{Sitio de estudio}

El ensayo se realizó en la Estación Experimental Agropecuaria Austral, perteneciente a la Universidad Austral de Chile, ubicada en Valdivia, Región de los Ríos, Chile $\left(39^{\circ} 47^{\prime}\right.$ Latitud Sur, $73^{\circ} 14^{\prime}$ Latitud Oeste), durante dos temporadas 2009/2010 y 2010/2011. Así mismo, los análisis fitopatológicos se llevaron a cabo en el Laboratorio de Fitopatología, del Instituto de Producción y Sanidad Vegetal, Facultad de Ciencias Agrarias, de la misma Universidad.

\section{Tratamientos y diseño experimental}

Ambos ensayos se realizaron con un diseño en parcelas subdividas y completamente aleatorizadas, con tres fuentes de variación, tres niveles y tres repeticiones por cada tratamiento. La Fecha de siembra ( $F 1=7 / 8 / 2009 ; F 2=1 / 9 / 2009$ y $F 3=22 / 9 / 2009$ para la primera temporada de estudio y $\mathrm{F} 1=8 / 8 / 2010$; $\mathrm{F} 2=1 / 9 / 2010$ y $\mathrm{F} 3=22 / 9 / 2010$ para el segundo período) fue considera como parcela principal, la densidad de siembra $\left(20,30\right.$ y 40 plantas $\left.\mathrm{m}^{-2}\right)$ fue considerada como subparcela y los cultivares utilizados en el estudio (Retaca, Alargá y Verde Bonita) fueron finalmente considerados como unidades de la sub-sub parcela. Así, la superficie de las parcelas fue de $30 \mathrm{~m}^{-2}(3 \times 10 \mathrm{~m})$, sub parcelas de $10 \mathrm{~m}^{-2}(1 \times 10 \mathrm{~m})$ y la sub-sub parcela de $3,3 \mathrm{~m}^{-2}(3,3 \times 1 \mathrm{~m})$ por cada repetición. Las evaluaciones fueron realizadas en 10 plantas al azar, por tratamiento y por repetición. El marco de plantación consideró una distancia entre hileras de $35 \mathrm{~cm}$ y 10, 8,3 y 7,1 cm sobre la hilera, para lograr de esta forma densidades de 20 , 30 y 40 plantas por $\mathrm{m}^{2}$. Las hileras de cada borde no fueron consideradas (efecto borde), por ello, no fueron incluidas en el análisis estadístico.

\section{Evaluación de enfermedades}

Para la evaluación de la manifestación de las distintas enfermedades, se realizaron estimaciones visuales de la sintomatología por fecha de siembra, cultivar y densidad. Estas estimaciones fueron realizadas una vez por semana, recolectando hojas con algún síntoma típico del ataque de patógenos, hasta el momento de cosecha, en donde se realizó la evaluación final para ver la incidencia de enfermedades para los distintos tratamientos. Para la identificación de los hongos del género Botrytis y Cercospora, se utilizaron características morfológicas descritas por Von Arx (1981); Morante (2007) y Mirzaei et al. (2008). Para el caso de $U$. viciae fabae, la identificación se realizó con las descripciones propuestas por Cummins (1978) y Emeran et al. (2005).

Para el diagnóstico de las enfermedades presentes en el material vegetal colectado en campo, se utilizó el protocolo que se detalla a continuación:

1. Cámaras húmedas a temperatura ambiente (18 a $22^{\circ} \mathrm{C}$ ) por 48 a $72 \mathrm{~h}$ y en paralelo siembras en medio de cultivo Agar Papa Dextroza + Ac. láctico al 2\% y Agar Malta.

2. Incubación de las placas sembradas en estufa a $20{ }^{\circ} \mathrm{C}+/-2{ }^{\circ} \mathrm{C}$, hasta 10 días.

3. Preparación microscópica de los signos de los hongos desarrollados en cámaras húmedas y en los medios de cultivo que presentaron esporulación.

4. Identificación con las claves descritas anteriormente.

La incidencia de patógenos (\%), se calculó en base a la siguiente formula:

$$
\text { Incidencia }(\%)=\frac{\text { Plantas enfermas }}{\text { Total de Plantas }} \times 100
$$

Además se obtuvo el rendimiento $\left(\mathrm{kg} \mathrm{ha}^{-1}\right)$, con el fin de relacionarlo a la incidencia de enfermedades. Los 
granos fueron secados a $60^{\circ} \mathrm{C}$ durante 48 horas en una estufa de secado para la obtención de peso seco.

\section{Análisis estadístico.}

Las variables de respuesta como porcentaje de incidencia de cada patógeno y rendimiento fueron sometidos a los supuestos de normalidad y homogeneidad de varianza (pruebas de Shapiro-Wilk y Levene). Sólo los datos de rendimiento cumplieron con dichos supuestos, por lo que fueron analizados mediante un ANOVA para representar las diferencias entre los tratamientos. Además, se realizó una separación de medias mediante la prueba de Tukey $(p<0,05)$. Por otra parte, la incidencia (\%) de patógenos, fue evaluada mediante la prueba de Kruskal-Wallis, y con una separación de medianas a posteriori mediante la prueba de Nemenyi $(p<0,05)$. Para el análisis de datos de rendimiento e incidencia de patógenos se utilizaron los programas SPSS Statistics 17.0 y StatSoft Statistica 7.0, respectivamente.

\section{RESULTADOS Y DISCUSIÓN}

\section{Presencia e incidencia de patógenos}

Durante las temporadas en que se realizó el estudio (2009/2010 y 2010/2011) los patógenos que se encontraron asociados al cultivo fueron: B. cinerea, B. fabae, causantes de la mancha chocolate del haba, $U$. viciae fa$b a e$, causante de la roya del haba, la cual fue diagnosticada solo durante la temporada 2009/2010, y C. zona$t a$, causante de la cercosporiosis, que solo se determinó durante la segunda temporada de cultivo. Los patógenos asociados al cultivo a excepción de $B$. cinerea, se encuentran descritos para el cultivo por Mujica y Vergara (1980).

$\mathrm{Al}$ analizar el efecto de los distintos tratamientos sobre la incidencia de patógenos se logró determinar para ambas temporadas que sólo la fecha de siembra presentó un efecto estadísticamente significativo $(p<0,05)$ sobre este parámetro. Durante la primera temporada $B$. fabae y U. viciae fabae, presentaron los valores más altos en la tercera fecha de siembra, en donde, se registraron valores sobre el $80 \%$ y $75 \%$ respectivamente. Para B. cinerea, la mayor presencia de este patógeno se determinó en la primera y segunda fecha de siembra (Figura 1).

Para la segunda temporada del cultivo (Figura 2) sólo $B$. fabae presentó diferencias estadísticamente significativas $(p<0,05)$ mostrando los valores más altos de incidencia en la primera y tercera fecha de siembra, en donde alcanzó un $62,2 \%$ y $70,4 \%$ de incidencia respectivamente. Durante esta temporada además se evidenció la presencia de $C$. zonata sin embargo, este patógeno solo se encontró en las plantas que fueron establecidas durante la primera fecha de siembra.

Estos resultados concuerdan con lo reportado por Hebblethwaite (1983); Marcellos y Constables (1986) y Sillero et al. (2010), quienes indican que siembras tempranas tendrían una gran influencia en la baja incidencia de B. fabae. Este patógeno es altamente dependiente de las condiciones ambientales, expandiéndose rápidamente en la planta y en el cultivo con temperaturas entre los 15 y $20^{\circ} \mathrm{C}$, con mínimas de 4 y máximas de $30^{\circ} \mathrm{C}$; además de presentar una relación directa con la humedad ambiental, distribuyéndose rápidamente sobre humedades relativas del 70\% (Hebblethwaite, 1983; Davison et al., 2007; Sillero et al., 2010), lo que

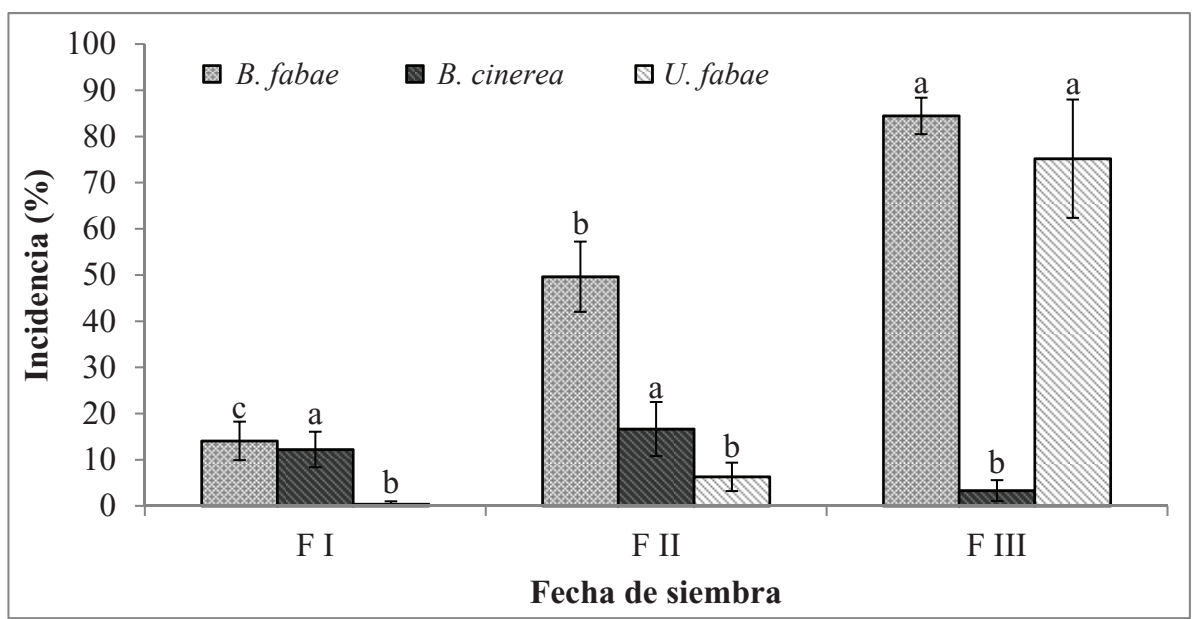

Diferentes letras en barras indican diferencias significativas entre patógenos $(p<0,05$, Nemenyi).

Figura 1. Incidencia de patógenos (\%), en distintas fechas de siembra (F1=7/8/2010; F2=1/9/2010 y F3=22/9/2010), temporada 2009/2010. (B. fabae: Botrytis fabae; B. cinerea: Botrytis cinerea; U. fabae: Uromyces fabae).

Figure 1. Pathogens incidence (\%) in different sowing date (F1=8/8/2010; F2=1/9/2010 y F3=22/9/2010), 2009/2010 season. (B. fabae: Botrytis fabae; B. cinerea: Botrytis cinerea; U. fabae: Uromyces fabae). 


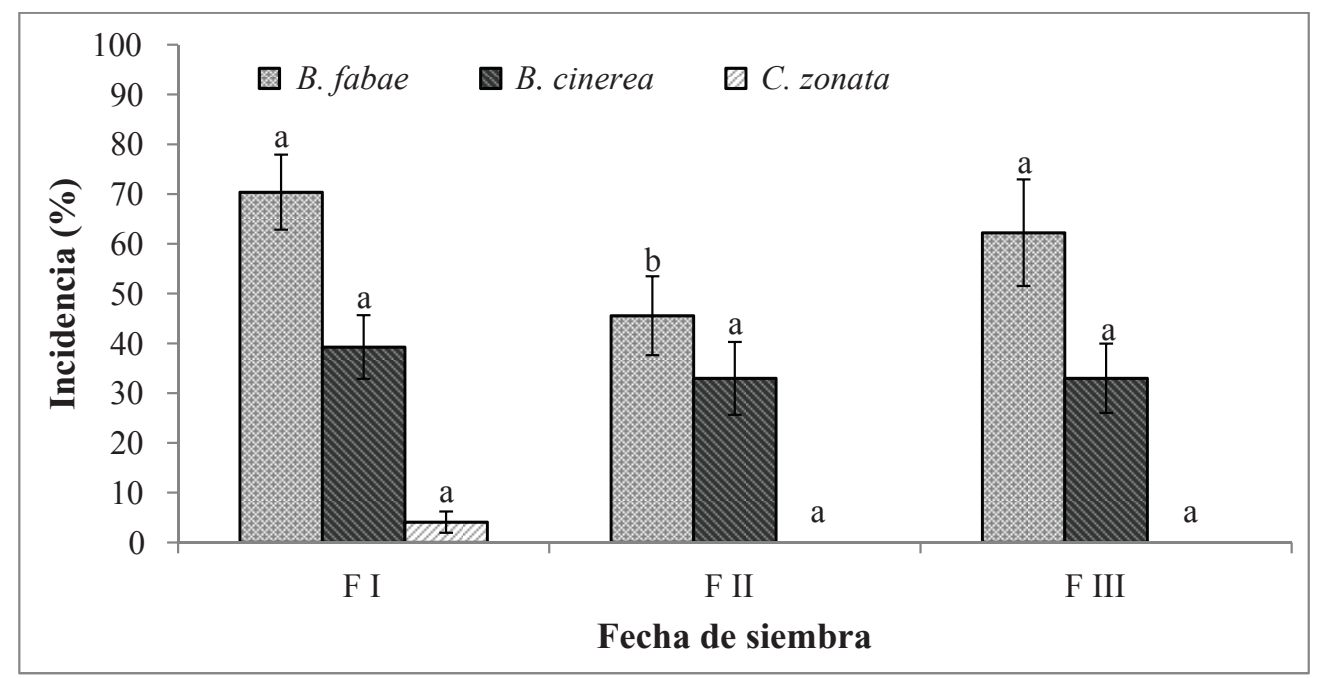

Diferentes letras en barras indican diferencias significativas entre patogenos $(p<0,05$, Nemenyi).

Figura 2. Incidencia de patógenos (\%), en distintas fechas de siembra (F1=8/8/2010; F2=1/9/2010 y F3=22/9/2010), temporada 2010/2011. (B. fabae: Botrytis fabae; B. cinerea: Botrytis cinerea; U. fabae: Uromyces fabae).

Figure2. Pathogens incidence (\%) in different sowing date (F1=8/8/2010; F2=1/9/2010 y F3=22/9/2010), 2010/2011 season. (B. fabae: Botrytis fabae; B. cinerea: Botrytis cinerea; U. fabae: Uromyces fabae).

explicaría el aumento de la presencia de este patógeno a medida que se atrasa la fecha de siembra, si bien esto es aplicable en su totalidad a la primera temporada de cultivo, durante el segundo periodo se observa que solo la fecha intermedia cumple con esto, lo que pudo haber sido ocasionado por las menores condiciones de humedad y temperatura que se presentaron previo a la emergencia de las plantas, lo que favoreció el desarrollo de este patógeno durante este periodo.

Los resultados obtenidos en este ensayo con respecto a $U$. viciae fabae para el primer año de estudio son similares a lo mencionado por Marcellos y Constables (1986) y Hanounik y Bisri (1991), ya que este patógeno se caracteriza por aparecer tarde en el cultivo, lo que se observó para las fechas uno y dos de siembra. Además, la alta incidencia de este patógeno para la tercera fecha, es explicada por las mejores condiciones de temperatura y humedad que favorecieron el desarrollo de este patógeno, el cual, presenta un crecimiento óptimo con temperaturas de $20^{\circ} \mathrm{C}$, esta condición fue lograda de mejor manera en la última fecha de siembra (Hanounik y Bisri, 1991).

Las fechas tempranas de siembra pueden evitar altos niveles de inóculo o las condiciones propicias para el desarrollo de una enfermedad en particular, por lo cual, existirían menores niveles de incidencia de patógenos (B. fabae y U. viciae fabae) al adelantar la fecha de siembra, esto debido principalmente a las menores temperaturas existentes en este período (Migawer y Bakeer, 2003).

Los valores más altos de presencia de patógenos para las fechas más tardías, podrían ser explicados ade- más, por el hecho de que al momento en que estos fueron evidenciados las plantas de las últimas siembras estaban en un estado menor de desarrollo, lo que determina un mayor tiempo a cosecha y por ende un mayor tiempo de diseminación del patógeno en el cultivo.

Los resultados provenientes de la presencia de enfermedades para las distintas densidades, no concuerdan por lo señalado en la literatura, la cual plantea que un incremento en la densidad de siembra se traduciría en un aumento en la incidencia de enfermedades (Agrios, 2005; Davidson et al., 2007; Sahile et al., 2008) ya que, una mayor población produciría un microclima en el interior del cultivo aumentando la humedad relativa y temperaturas. A medida que el número de plantas en un cultivo es mayor, la competencia entre individuos aumenta, razón por la cual las plantas tienden a generar un menor número de estructuras con el fin de optimizar los recursos (Briones, 2009). Datos publicados por Briones (2009), señalan que cultivares de haba de crecimiento determinado que fueron sometidos a altas densidades de siembra, tendieron a producir un número menor de ramas por planta, lo que podría explicar que no existan diferencias entre la incidencia de enfermedades para las distintas densidades, ya que, las plantas sometidas a una menor densidad producirían una mayor cantidad de tallos, produciéndose así un clima al interior del cultivo similar al obtenido con altas densidades de siembra (menor número de tallos). Otro factor a considerar es que los cultivares de crecimiento determinado tienen una altura que no supera los $60 \mathrm{~cm}$, lo que mejoraría en cierta forma la aireación del cultivo. 


\section{Rendimiento}

Durante la primera temporada de cultivo se determinó un efecto estadísticamente significativo $(p<0,05)$ entre las fechas de siembra, mostrando los mayores rendimientos la primera y segunda fecha de siembra (7 de agosto y 1 de septiembre), la menor productividad del cultivo fue observada en la última fecha de siembra (22 de septiembre de 2009) (Cuadro 1). Al analizar los efectos de la densidad de plantas sobre el rendimiento, se observaron diferencias significativas $(p<0,05)$, en donde los rendimientos más altos fueron registrados por las mayores densidades ( 30 y 40 plantas $\mathrm{m}^{-2}$ ). Así mismo, no se presentaron diferencias estadísticamente significativas entre cultivares $(p<0,05)$, presentando en promedio un rendimiento de 3200 a $3600 \mathrm{~kg} \mathrm{ha}^{-1}$ de haba.

En la segunda temporada de cultivo se observó un efecto estadísticamente significativo $(p<0,05)$ para los factores fechas de siembra y densidad, obteniéndose los mayor rendimientos en la segunda fecha de siembra (1 de septiembre) y con la densidad de 40 plantas $\mathrm{m}^{-2}$ (Cuadro 2), en donde se obtuvo un promedio de $3000 \mathrm{~kg} \mathrm{ha}^{-1}$ para ambas variables. No se observaron diferencias estadísticamente significativas entre los distintos cultivares utilizados.

El mismo efecto sobre el rendimiento de la fecha y densidad de siembra ha sido descrito por Marcellos y Constables (1986); Saxena et al. (1991); Turk y Tawaja (2002); López-Bellido et al. (2005); Nadal y Moreno (2006) y Briones (2009), que reportaron un efecto positivo en el rendimiento gatillado por fechas tempranas de siembra y altas densidades de plantas.

Cuadro 1. Rendimientos $\left(\mathrm{kg} \mathrm{ha}^{-1}\right)$, por fecha de siembra, densidad y cultivar, para la temporada 2009/2010.

Table 1. Yields $\left(\mathrm{kg} \mathrm{ha}^{-1}\right)$ according to sowing date, plant density and cultivar, 2009/2010 season.

\begin{tabular}{|c|c|c|}
\hline \multicolumn{3}{|c|}{ Rendimiento (kg ha-1) } \\
\hline \multicolumn{3}{|c|}{ Fecha de siembra } \\
\hline 7 de agosto & 1 de septiembre & 22 de septiembre \\
\hline $3800 \mathrm{a}$ & $3600 \mathrm{a}$ & $2800 \mathrm{~b}$ \\
\hline \multicolumn{3}{|c|}{ Densidad (plantas $\mathrm{m}^{-2}$ ) } \\
\hline 20 & 30 & 40 \\
\hline $2600 \mathrm{~b}$ & $3600 \mathrm{a}$ & $4100 \mathrm{a}$ \\
\hline \multicolumn{3}{|c|}{ Cultivar } \\
\hline Alargá & Retaca & Verde Bonita \\
\hline $3600 \mathrm{a}$ & $3400 \mathrm{a}$ & $3200 \mathrm{a}$ \\
\hline
\end{tabular}

Diferentes letras en las filas indican diferencias significativas $(p<0,05$, Tukey).
La evaluación de incidencia de enfermedades para los distintos tratamientos demostró que siembras tempranas ( 7 de agosto y 22 de septiembre de 2010 y 22 de septiembre de 2011) presentan los valores más bajo de incidencia para las enfermedades en general. Por otra parte, la última fecha de siembra es la que muestra la mayor presencia de enfermedades. Al analizar los resultados de rendimiento e incidencia, se puede apreciar una relación inversa entre estos factores, es decir, a medida que la incidencia de enfermedades aumenta el rendimiento se reduce (Cuadro 1), por lo que al manejar estas variables agronómicas sería posible maximizar los rendimientos y minimizar la incidencia de patógenos, aumentando de esta forma los ingresos a nivel de productor. Marcellos y Constables (1986); Saxena et al. (1991); Turk y Tawaja (2002); López-Bellido et al. (2005) señalan que los mayores rendimientos obtenidos en fechas más tempranas de siembra se atribuyen principalmente a una mayor duración de la etapa de crecimiento vegetativo y llenado de grano, lo que determina una mayor productividad del cultivo.

\section{CONCLUSIONES}

Siembras en fechas tardías presentaron una mayor incidencia de enfermedades, independiente de las densidades de siembra o cultivares que fueron utilizados en este estudio.

Fechas de siembra temprana y altas densidades incrementan el rendimiento esperado, independiente de los cultivares utilizados.

Cuadro 2. Rendimientos ( $\mathrm{kg} \mathrm{ha}^{-1}$ ), por fecha de siembra, densidad y cultivar, para la temporada 2010/2011

Table 2. Yields $\left(\mathrm{kg} \mathrm{ha}^{-1}\right)$ according to sowing date, plant density and cultivar, 2010/2011 season.

\begin{tabular}{|c|c|c|}
\hline \multicolumn{3}{|c|}{ Rendimiento (kg ha ${ }^{-1}$ ) } \\
\hline \multicolumn{3}{|c|}{ Fecha de siembra } \\
\hline 8 de agosto & 1 de septiembre & 22 de septiembre \\
\hline $2100 \mathrm{~b}$ & $3000 \mathrm{a}$ & $2200 \mathrm{~b}$ \\
\hline \multicolumn{3}{|c|}{ Densidad (plantas $\mathrm{m}^{-2}$ ) } \\
\hline 20 & 30 & 40 \\
\hline $1800 \mathrm{c}$ & $2500 \mathrm{~b}$ & 3000 a \\
\hline \multicolumn{3}{|c|}{ Cultivar } \\
\hline Alargá & Retaca & Verde Bonita \\
\hline $2400 \mathrm{a}$ & $2400 \mathrm{a}$ & $2400 \mathrm{a}$ \\
\hline
\end{tabular}

Diferentes letras en las filas indican diferencias significativas $(p<0,05$, Tukey). 


\section{AGRADECIMIENTOS}

Los autores agradecen el soporte financiero de la Dirección de Investigación de la Universidad Austral de Chile (Proyecto DID UACH S-2009-68).

\section{REFERENCIAS}

Agrios, G., 2005. Plant Pathology. 5a ed. Academic Press, San Diego.

Amira, A., Fallal, E., Migahed, F., 2003. Metabolic changes in broad bean infected by Botrytits fabae in response mushroom spent straw. Asian Journal of Plant Science 2, 10591068. http://dx.doi.org/10.3923/ajps.2003.1059.1068.

Briones, Y., 2009. Evaluación de dos cultivares de haba tipo "baby" (Vicia faba L.) bajo diferentes poblaciones para la industria de congelado. Tesis Lic. Agr. Santiago. Universidad de Chile. 59 p.

Cummins, G., 1978. Rust fungi, on legumes and composites in North America. The University of Arizona Press, Arizona.

Davidson, J., Pande, S., Bretag, T., Lindbeck, K., Kishore, G., 2007. Biology and management of Botrytis spp. in legume crops, in: Elad, Y., Williamson, B., Tudzynski, P., Denle, N., (Eds.), Botrytis biology, pathology and control. Springer, Dordrecht, pp. 295-318.

Elad, Y., Williamson, B., Tudzynski, P., Denle, N., 2007. Botrytis: Biology, Pathology and Control. Springer, Dordrecht.

Emeran, A., Sillero, J., Niks, R., Rubilaes, D., 2005. Infection structures of host specialized isolates of Uromyces viciae fabae and of other species of Uromyces infecting leguminous crops. Plant Disease 89, 17-22. <http://dx.doi. org/10.1094/PD-89-0017>.

Faiguenbaum, H., 2003. Labranza, siembra y producción de los principales cultivos de Chile. Vivaldi, Santiago.

Hebblethwaite, P., 1983. The faba bean (Vicia faba L.). Scribe Design, Cambridge.

Hanounik, S., Bisri, M., 1991. Status of diseases of faba bean in the mediterranean region and their control. Options Méditerranéennes 10, 56-66.

López-Bellido, F., López-Bellido L., López-Bellido R. 2005. Competition, growth and yield of faba bean (Vicia faba L.). European Journal Agronomy 23, 359-378. http:// dx.doi.org/10.1016/j.eja.2005.02.002.

Marcellos, H., Constables, G., 1986. Effects of plant density and sowing date on grain yield of faba beans (Vicia faba L.) in northern New South Wales. Australian Journal of
Experimental Agriculture 26, 493-496. <http://dx.doi. org/10.1071/EA9860493>.

Migawer, E., Bakeer, A., 2003. Influence of sowing dates, plant spacings, cultivars and their interactions on wilt disease incidence, seed yield and yield components of lupins. Departamento de Agronomía, departamento de Botánica Agrícola. El Cairo. Universidad de Fayoum. 17p.

Mirzaei S., Mohammadi Goltapeh, E., Shams-Bakhsh, M., Safaie, N., 2008. Identification of Botrytis spp. On Plants Grown in Iran. Journal of Phytopathology 156, 21-28. http://dx.doi.org/10.1111/j.1439-0434.2007.01317.x.

Morante, M., 2007. Manchas foliares del haba (Vicia faba L.). Boletín técnico, Universidad Mayor de San Simón. Facultad de Ciencias Agrícolas, Pecuarias, Forestales y Veterinarias Dr. Martín Cárdenas. Cochabamba.

Mujica, F., Vergara, C., 1980. Flora fungosa chilena. Segunda Edición. Universidad de Chile. Facultad de Agronomía. Santiago.

Nadal, S., Moreno, M., Cubero, J., 2004. Las leguminosas grano en la agricultura moderna. Mundi Prensa, Madrid.

Nadal, S., Moreno, M., 2006. Optimal population density of determinate growth habit faba bean for inmature green pod production. Agriculture Conspectus Scientificus 71, 37-39.

Sahile, S., Ahmed, S., Fininsa, C., Abang, M., Sakhuja, K., 2008. Survey of chocolate spot (Botrytis fabae) disease of faba bean (Vicia faba L.) and assessment of factors influencing disease epidemics in northern Ethiopia. Crop Protection 27, 1457-1463. <http://dx.doi.org/10.1016/j. cropro.2008.07.011>.

Saxena, M., Silim, S., Matar, A., 1991. Agronomic management of faba bean for high yields. Options Méditerranéennes 10, 91-96.

Sillero, J., Villegas, A., Thomas, J., Rojas, M., Emeran, A., Fernandez, M., Rubilaes, D., 2010. Faba bean breeding for disease resistance. Fields Crops 115, 297-307. http:// dx.doi.org/10.1016/j.fcr.2009.09.012.

Stoddard F., Nicholas, A., Rubilaes, D., Thomas, J., Villegas, A., 2010. Integrated pest management in faba bean. Field Crops 115, 308-318. <http://dx.doi.org/10.1016/j. fcr.2009.07.002>.

Turk, M., Tawaja, A., 2002. Impact of seeding rate, seeding date, rate and method of phosphorus application in faba bean (Vicia faba L. minor) in the absence of moisture stress. Biotechnology, Agronomy, Society and Environment 6, 171-178.

Von Arx, J., 1981. The genera of fungi sporulating in pure culture. Gantner Verlag, Vaduz. 